\title{
Entretien
}

\section{Pierre Dansereau, le gentilhomme décodeur et iconoclaste de l'écologie}

\section{Propos recueillis par Normand Brunet et Agnès Pivot}

\author{
Pierre Dansereau ${ }^{\mathrm{a}}$, Normand Brunet ${ }^{\mathrm{b}, \star}$, Agnès Pivot $^{\mathrm{c}, \star, \star \star}$ \\ a Écologue, Laboratoire Pierre Dansereau pour l'étude des écosystèmes, Université du Québec à Montréal, Case postale 8888, \\ succursale centre-ville, Montréal (Québec), H3C 3P8, Canada \\ b Biologiste, Chaire d'études sur les écosystèmes urbains, Institut des sciences de l'environnement, Université du Québec \\ à Montréal, Case postale 8888, succursale centre-ville, Montréal (Québec), H3C 3P8, Canada \\ c Sociologue, Ladyss/NSS, Maison Max Weber, Université Paris X, 200 av. de la République, 92001 Nanterre cedex, France
}

"Il faut affronter le froid et la misère, se mouiller les pieds et respirer l'air pollué pour incarner une véritable problématique environnementale. » P. Dansereau ${ }^{1}$

Agnès Pivot / Normand Brunet : Pierre Dansereau, vous êtes un homme surprenant; précurseur de la démarche interdisciplinaire, vous parlez de votre travail scientifique en termes littéraires et engagés. Tout d'abord, parlons de l'importance pour votre parcours professionnel du voyage et des rencontres, Pour vous, écologue, on peut dire que le terrain c'est « quitter l'érablière laurentienne, premier foyer de mes recherches pour remonter à la source équatoriale, puis osciller entre les pôles pour reconstruire la gamme planétaire des contraires climatiques ». Vous avez aussi ajouté : «Une déférence à l'expérience des autres aura toujours accompagné la révélation que $m^{\prime}$ apportait chaque paysage... Le voyage est ma démarche la plus naturelle, la solidarité la valeur la plus chère ${ }^{2}$.» Pouvez-vous tout d'abord nous dire quelles personnalités, aussi bien scientifiques que littéraires ou culturelles, vous ont accompagné dans ce parcours?

Pierre Dansereau : Il faut remonter assez loin, ça commence avec la littérature, pas avec la science. À 16 ans,

\footnotetext{
* Cet entretien a été réalisé le 8 mai 2002. Ce texte fait partie d'un projet de numéro spécial NSS élaboré sous la direction de Laurent Lepage (Chaire d'études sur les écosystèmes urbains de l’Université du Québec à Montréal) qui doit paraître en 2004.

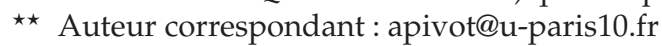

1 1991. L'Envers et l'endroit : le besoin, le désir et la capacité, Québec, Musée de la Civilisation, p. 19.

2 1993. Je n'ai jamais été dépaysé. Revue Liberté, 208-209, 22-23.
}

j'avais une grande amie écrivain et journaliste qui en avait 72. Elle m'a appris à écrire. L'été, à Percé en Gaspésie, j'écrivais des romans, des vers, j'étais pris de la frénésie de l'écriture; elle corrigeait mes textes, elle se moquait gentiment de moi.

Dans ces années adolescentes, j'ai vécu huit ans de purgatoire chez les Jésuites - un système d'éducation où la discipline primait toujours sur l'intellect, à une exception près : en année de belles-lettres, mon professeur, le père Georges-Henri d'Auteuil, m'a dit : « Et vous, qu'estce que vous pensez de telle chose? » Un professeur qui me demandait ce que moi je pensais! Qui suis-je pour penser? Je le savais déjà ! J'avais la conviction que j'avais quelque chose à dire. Le père d'Auteuil nous donnait des devoirs à faire en fin de semaine et nous passions des heures enfermés dans notre chambre à écrire 40 pages sur des thèmes comme Claudel a dit : «Le mal ne compose pas, est-ce vrai ? » ou bien : «Que peut-on faire avec une goutte d'eau? »C'était un professeur vraiment présent, ouvert à l'échange et il lisait en classe les meilleurs papiers. Il y avait une certaine émulation et un bonheur extraordinaire à être lu ainsi. Il les lisait tout haut sur un ton très modulé; de temps en temps quand on exagérait un peu, il levait les sourcils et il disait : "Ah! un mot qui est bien choisi, bien trouvé, un vocabulaire en voie d'enrichissement... » Une expérience extraordinaire!

Le père d'Auteuil m'a énormément influencé. Vingt ans plus tard j'ai adopté une méthode pédagogique similaire vis-à-vis de mes étudiants, les faire parler, et leur dire : «Si je n'apprends rien de vous, vous n'apprendrez 
pas grand-chose de moi. » Ces rencontres diachroniques, dans l'espace réel, dans le temps réel où l'on vit, ont toujours été très importantes pour moi et maintenant c'est moi qui suis à l'autre bout du spectre!

Il y a eu aussi Albert Pelletier, notre meilleur critique littéraire dans le temps ; il a bien voulu croire que j'avais, disait-il, des idées. Il a publié une revue qui a duré une dizaine d'années et qui s'appelait justement Les Idées. Il était à la fois accueillant et sévère.

Cette acquisition d'un instrument d'écriture et de paroles, lorsque j'ai fait le virage à la science, m'a été d'une utilité extraordinaire. À l'université de Michigan où j'ai enseigné à partir de 1950, les étudiants et mes collègues valorisaient énormément la culture générale que j'avais. Il faut dire que j'étais bilingue. Bilingue depuis la plus tendre enfance, ma mère étant franco-américaine, on parlait souvent l'anglais à la maison. Il s'est trouvé par hasard que les enfants du voisinage étaient tous ou presque tous anglophones. Dans ce milieu scientifique des ÉtatsUnis, nombreux étaient les collègues américains qui enviaient ma facilité d'expression. Eux en étaient aux éprouvettes et aux calculs, et aux tableaux, et aux graphiques, mais ils étaient - plusieurs d'entre eux - très peu maîtres de la langue anglaise et il m'est arrivé souvent de les aider de ce côté-là.

De plus, je dessinais assez bien et ma femme, une artiste peintre, a fait mon éducation sur le plan esthétique. De 1936 à 1939, nous avons visité les musées en France. Cet enrichissement progressif m'a encouragé dans l'idée de me servir de tous mes moyens, quoi que je fasse, écrire un poème ou résoudre un problème scientifique.

Les influences plus «scientifiques » maintenant. Cela a démarré avec le frère Marie Victorin, botaniste, qui comme moi a été littéraire avant d'être scientifique. Je me suis orienté vers l'agronomie, avec l'idée de vivre sur une ferme, de pratiquer l'agriculture et de faire de la politique. Je passe rapidement sur l'épisode politique où je m'étais identifié avec un mouvement anti-partisan, anti-libéral, anti-conservateur. J'ai passé trois ans en Europe et je ne suis pas resté dans l'horticulture, je me suis embarqué complètement dans la botanique, et dans la botanique pure, à la suite en somme de Marie Victorin, qui m'encourageait non pas à faire comme lui, mais à prolonger son œuvre dans un autre domaine. L'écologie existait si peu qu'il n'y avait pas d'universités françaises qui l'admettaient. Le professeur Braun-Blanquet la pratiquait à la Station internationale de géo-botanique méditerranéenne et alpine - SIGMA ; mais il n'était pas reconnu, il n'avait pas le droit de prendre des étudiants en doctorat.

Alors, j'ai fait une thèse à Genève en botanique, et j'ai eu là un maître qui était dans la belle tradition genevoise, très agréable et très utile. Mais, moi, ce qui m'intéressait, c'était l'écologie et j'allais rejoindre monsieur Braun-Blanquet dans les Alpes, dans les Pyrénées; on faisait beaucoup d'excursions et j'ai appris sa méthodologie, que j'ai tout de suite adaptée à des fonctions différentes de ce qu'on proposait à Montpellier. Au cours de ces études de botanique, je me suis familiarisé avec Darwin, Humboldt, le fondateur de la biogéographie. Ses œuvres, son aventure tropicale, m'ont hanté et je l'ai retrouvé en 1945 quand je suis allé au Brésil. Darwin, sur le plan social, sur le plan religieux, m'apportait quelque chose d'assez nouveau et d'immédiatement assimilable à ma culture classique. Darwin, Wallace et Huxley, le grand-père Huxley, tous me parlaient un langage que je pouvais comprendre et appliquer dans mes travaux.

De retour à Montréal, un mois avant la déclaration de guerre en 1939, je reçois tous les octrois que je voulais du Conseil national de recherche à Ottawa pour faire de la phytosociologie de l'érablière. La sociologie végétale n'était pas dans le vocabulaire des universitaires canadiens. Â Chicago, dans le Nebraska, dans le Middle West américain, sauf Harvard et la Californie qui eux disaient non à l'écologie sous toutes ses formes. Je me trouvais ainsi sur une marge du progrès scientifique mais potentiellement en position d'inventer quelque chose.

Ma plus grande préoccupation, pendant les années 1940 et 1950, était l'excès d'intérêt donné à la composition - la présence de telle ou telle espèce, qui est un indicateur d'humidité, d'acidité et de bien d'autres choses - et pas assez de description de la biomasse, de la masse végétale. Qu'est-ce qu'une forêt? Je me suis aperçu que personne ne pouvait définir ce qu'était une forêt; c'est d'abord un arbre, or on ne savait pas ce qu'était un arbre et on ne le sait toujours pas, il n'y a pas de définition scientifique. Une forêt d'érables, une forêt de chênes, une forêt de sapins, ce sont des assemblages et des biomasses complètement différents par leur texture, par leur forme, par leur fonction. Alors, j'ai proposé un répertoire de formes et de fonctions, d'associations de stratifications, de périodicités, qui nous donne une meilleure chance d'évaluer, de quantifier le potentiel d'une forêt d'érables comparée à une forêt de sapins ou de pins.

\section{A.P. : Cela a donné l'ouvrage Biogeography en $1957^{3}$ ?}

Pierre Dansereau : Oui. Biogeography a été le compendium en quelque sorte de mes acquisitions et de mes petites inventions, C'était le seul traité de biogéographie qui faisait une place à l'homme, «naturel » en ce temps-là signifiant «absence de l'homme ». Moi, évidemment, je n'acceptais pas cette limitation. Je me demandais : «Estce que tout ce que l'on a découvert sur le dynamisme des écosystèmes dits "naturels" s'applique à des espaces de pâturages, à des espaces agricoles, industriels, urbains, inter-spatiaux? » Pour moi c'était : «Oui. »

\footnotetext{
3 Dansereau, P., 1957. Biogeography: an ecological perspective, New-York, Ronald Press.
} 


\section{N.B. : Comment s'est passée cette transition?}

Pierre Dansereau : J'ai publié en 1965 un article important - pour moi, en tout cas - à l'occasion d'un colloque exemplaire, organisé par la Conservation Foundation aux USA, par Frank Fraser Darling. On nous avait recrutés un an et demi, presque deux ans d'avance, avec énormément de préparation écrite et d'échanges au préalable. Le moment venu, j'ai fait un boniment assez étoffé sur l'écologie humaine en m'attaquant à la question suivante : "L'arsenal de lois et de processus que nous avons accumulés sous l'égide de l'écologie par l'étude des plantes et des animaux vivant à l'état sauvage, peutelle être transférée à l'Homme? Est-ce qu'on peut parler dans le même souffle de compétition-coopération, de succession, etc. ? » Il y avait longtemps que je faisais cela, avec mes étudiants. La succession, par exemple - un quartier riche qui devient un quartier pauvre, un quartier pauvre qui s'embourgeoise -, est un processus qui existe aussi dans la nature, et pour des causes identiques, ou tout au moins analogues, et certainement parallèles.

Cette conversion a été accentuée par ma nomination à New York en 1961, comme sous-directeur du Jardin botanique de New York. J'assistais assez souvent à des réunions du conseil d'administration, lequel se composait des millionnaires de Wall Street et de leurs femmes de Park Avenue. À l'occasion d'un symposium sur les fleurs de 1'Amérique du Nord, elles ont invité à déjeuner les scientifiques et une fois sortis sur le balcon, je leur ai dit: «Mesdames, les fleurs, on ne les voit pas. Qu'est-ce qu'on voit? La pollution, la criminalité, le changement social, une écologie désastreuse... » et j'ai réussi à les convaincre de laisser tomber les fleurs, qui étaient en bonnes mains par ailleurs, pour organiser un colloque réunissant des écologistes de toutes sortes - microbiologistes, forestiers ou autres - et des présidents ou viceprésidents de compagnies, des directeurs de musées, des politiciens, des décideurs de tout acabit, dans un esprit, non pas de confrontation mais de collaboration. Il s'agissait de voir quel était l'état de la terre, de l'air, de l'eau dans la mégalopole. On a publié un livre, Challenge for survival. Cette initiative m'a réellement convaincu que je devais étudier les applications, les méthodes, les processus de l'écologie tels qu'ils se révèlent dans la vie animale, végétale mais aussi humaine.

C'est à cette époque que j'ai été rappelé à Montréal pour l'étude de faisabilité de l'aéroport international de Mirabel.

A.P. : À cette occasion vous étiez le coordinateur d'une équipe de psychologues, de sociologues, d'agronomes... Comment avez-vous fait pour les faire travailler ensemble?

Pierre Dansereau : Les ingénieurs, qui ne connaissaient pas les tourbières, avaient déjà massacré des dunes magnifiques lorsque le gouvernement fédéral a décidé de faire appel à d'autres compétences. J'étais revenu non pas à mon ancienne faculté dont j'avais été doyen, mais à la nouvelle faculté de l'Aménagement, à l'institut d'Urbanisme. Je suis allé chercher certains de mes anciens élèves un peu partout pour leur demander s'ils voulaient accepter de faire ce travail-là. Il y avait donc des géologues, des climatologues, des botanistes, des zoologistes, en particulier des ornithologues parce que le péril aviaire était quelque chose de très important, mais aussi des géographes et des ingénieurs, des psychologues et des sociologues.

Nous nous consultions constamment sur le terrain. Par exemple, le botaniste aurait pu, dans sa logique de recherche, vouloir un inventaire complet de la flore, avant de s'occuper de la végétation, mais nous on voulait qu'il aille tout droit à la végétation, et non pas à la flore. De la même façon, les ornithologues devaient observer le triangle de parcours quotidien (nidification, alimentation et dortoir), et si cela passait près du trajet proposé pour les atterrissages, détruire ou modifier l'un ou l'autre de ces endroits, afin de les empêcher de dormir ici, de manger là...

A.P. : Votre manière de travailler, c'était de vous rencontrer souvent?

Pierre Dansereau : Très souvent, je ne dis pas que nous avons eu un succès total dans les concessions que chacun devait faire. Il y a eu des moments un peu durs où on allait même jusqu'à s'engueuler passablement entre géographes et zoologistes, par exemple... Quant à moi, je n'ai pas d'autorité - je suis un anarchiste et je l'ai découvert seulement à 54 ans, je reçois mal et je n'exerce pas du tout l'autorité - donc je n'ai pas été un coordinateur très ferme qui serre la vis et qui dit «non, Roger tu ne peux pas faire cela »... J'ai été un superviseur, un coordinateur, un pacificateur, mais je ne réclame pas un succès sensationnel pour cette expérience.

A.P. : Alors, c'est peut-être pour cela que ça a marché...

Pierre Dansereau : Ça a marché assez bien. Il y avait trois choses qui nous préoccupaient. Premièrement, corriger des erreurs, ce qui a été très difficile, les contrats ayant déjà été signés, il y avait peu de choses que nous pouvions rectifier - les dunes par exemple, porteuses potentielles d'un très beau projet récréatif, avaient déjà été dévastées...

Deuxièmement, inventer une méthodologie qui n'existait pas encore à ce moment-là, non pas avec l'idée que ce serait la méthodologie mais une expérience à faire, une interdisciplinarité à créer entre gens qui se rencontrent, se retrouvent sous la pluie, dans la neige, dans un beau décor, un bon repas ensemble...

J'ai donc élaboré une boule de flèches, qui est une conception de l'écosystème beaucoup plus générale que 
celle dont on disposait jusqu'ici, basée uniquement sur le circuit alimentaire. Elle ajoute d'autres dimensions et comme un écosystème, elle comporte des niveaux trophiques : le niveau minéral, qui nourrit le niveau végétal, le niveau végétal qui nourrit les herbivores, les carnivores qui se nourrissent d'herbivores, et finalement deux dimensions nouvelles dans ce domaine, l'investissement qui part du nid de l'oiseau pour aller jusqu'au compte en banque, et le dernier niveau, le contrôle. Avec la possibilité de circulation de ressources et d'un input et d'un output. Elle n'a pas fait trois fois le tour du monde. Il y a deux personnes au Japon et trois personnes en Australie et une autre en Italie qui l'ont utilisée. Ça n'a pas été un succès mondial, je ne me fais pas d'illusions là-dessus, mais je pense qu'on a avancé d'un pas dans la relation des académiciens avec les pouvoirs gouvernementaux et les pouvoirs financiers. Ce qui m'amène à la troisième chose dont je voulais parler.

Lors de cette expérience, les universités ne nous ont pas beaucoup aidés et l'interdisciplinarité s'est pratiquée en dehors d'elles, à la Baie James, à Mirabel, dans la planification urbaine, par des professeurs des différentes universités qui se rencontraient sur le terrain. Comme je me plais à le répéter, les cloisons qui séparent les départements à l'université sont plus épaisses que les cloisons extérieures de l'université.

N.B. : Je voulais justement vous amener sur le terrain des cloisons et des murs qui existent entre les départements universitaires, et entre l'université et la société. J'allais formuler quelque chose qui vous qualifiait de modèle de décloisonnement, dans le sens où vous avez fait des rapprochements entre la littérature scientifique et la littérature en soi, entre les arts et la science, entre la ville, le milieu naturel, le milieu rural, etc. Vous avez constamment cherché à travailler sur les interfaces, donc l'interdisciplinarité allait de soi. Pourquoi est-ce si difficile de travailler dans une perspective horizontale et d'ouvrir des brèches dans ces murs, ou dans ces cloisons qui sont si épaisses, pour favoriser un genre de libre-échange des idées ou des modèles, pour agir au niveau horizontal?

Pierre Dansereau : Il y aurait plusieurs réponses à donner. Celle qui est peut-être la plus évidente, c'est l'impossibilité d'obtenir des subsides par les organismes donateurs pour tout ce qui excède une discipline. J'ai siégé pour la réforme du Conseil national de recherche à Ottawa, en 1978, où l'on a fait une refonte des organismes subventionnaires. Il y avait le Conseil national de recherche médicale, le Conseil national de science et d'ingénierie, et le Conseil national des arts et des sciences humaines. J'ai siégé dans le premier groupe de direction, d'administration du Conseil national de recherche en science et ingénierie. Et les présidents des deux autres organismes étaient présents. Parce qu'on avait dit qu'il faudrait faire de l'interdisciplinarité. Ça n'a pas marché, mais pas du tout.

J'ai moi-même présenté quelques années plus tard un projet de cartographie multidimensionnelle qui couvre toute la cascade de l'influence de l'homme sur le paysage. Mais cela a été refusé à Ottawa parce que, pour le Conseil national de recherche en science, ce n'était pas assez "scientifique», pour l'autre ce n'était pas assez «humain».

J'étais entre deux chaises et pour beaucoup de gens qui, comme moi, ont voulu faire non pas de la multi mais de l'interdisciplinarité, il y a eu des échecs et il continue d'y en avoir. Ici, par exemple, à l'Institut des sciences de l'environnement, nous sommes très étoffés en personnel issu de la constellation des sciences de l'environnement, chacun dans son domaine bien délimité, apte à donner des équations biochimiques et des choses comme cela pour définir leur projet, et c'est tout de suite dans le bon créneau comme on dit. Moi, j'ai toujours été dans un mauvais créneau...

A.P. : Vous dites de vous-même «je suis un décodeur». Pourquoi?

Pierre Dansereau : Je suis un décodeur car, en tant que naturaliste, je pose des questions et je trouve de temps en temps des réponses. La question que je me suis posée dans les premières années de ma recherche, dans les années 1940, était: «Quels sont les mécanismes qui assurent la succession des plantes sur un terrain nu, sableux par exemple? Quelles sont les premières plantes qui vont envahir et pourquoi ne peuvent-elles pas y demeurer très longtemps? » C'est ainsi que j'ai décodé un peu de l'ensemble de la végétation du Québec méridional et de l'Arctique, je suis allé en Nouvelle-Zélande et au Brésil, et ailleurs où j'ai appliqué ces principes-là. J'ai détecté des successions analogues, même si elles impliquaient des plantes qui n'ont aucune parenté les unes avec les autres.

Mais oui, je suis décidément un décodeur. En m'appliquant à des espaces occupés et transformés par l'Homme, est-ce que je peux rejoindre les séquences de l'érablière et retrouver dans l'espèce humaine, comme chez les espèces végétales et animales, la même combinaison de coopération et de compétition, à l'intérieur d'une espèce, ou entre espèces? Et des espèces qui peuvent transgresser plusieurs étages dans l'espace, et d'autres qui sont limitées à une strate. Est-ce que c'est le manque d'eau ou la présence d'un autre organisme qui empêche une espèce d'être représentée par beaucoup d'individus, alors que d'autres ont des centaines d'individus?

A.P. : Pour revenir à la science et à la préoccupation plus spécifique de NSS, pourriez-vous parler de votre espérance de « réconciliation » entre ce que vous décrivez comme deux solitudes, à savoir celle des sciences biologiques et celle des sciences humaines? 
Pierre Dansereau : Je ne suis pas très optimiste, la réconciliation de ce qu'on a appelé «les deux cultures » ne s'est pas faite. Il y a des secteurs importants de la population où la question ne se pose même pas. C'est le cas des gens qui ont un petit métier à faire, une envergure limitée et qui ne se demandent pas s'ils devraient être mieux renseignés au point de vue scientifique ou plus articulés dans la façon d'en parler.

De plus en plus, on fait des schémas, des soi-disant «modèles ». Des modèles, il paraît que j'en ai fait un. Moi, j'avais l'impression de faire un dessin. Comme je vous l'ai dit, j'ai été exposé, par ma femme et par mon milieu, à la peinture abstraite et mon parcours scientifique n'aurait pas été le même si je n'avais pas été frappé, et même enchanté par elle. Quand on peut parler un peu les deux langues, quand on peut assimiler à la science des perceptions poétiques... J'ai travaillé avec René Derouin, un sculpteur-peintre qui a organisé plusieurs colloques dans sa magnifique propriété dans les Laurentides. Il a illustré mon texte avec des peintures murales, et c'était très approprié.

A.P. : J'aimerais revenir sur le terrain car j'aime ce que vous en dites, cette double passion, à la fois pour la nature et pour les hommes qui sont pour vous indissociables et qui s'aborde avant tout par les sens. Vous avez dit : « Le temps est sûrement venu pour une réconciliation des sciences naturelles et des sciences de l'homme et en fait pour une reconnaissance de la découverte authentique par les moyens artistiques aussi bien que scientifiques et explicitement rationnels. Le dévoilement du processus de l'apprentissage lui-même et des ouvertures émotionnelles dans le cheminement de l'investigation scientifique jette un éclairage indispensable à la compréhension de toutes les découvertes, grandes et petites $^{4}$.»

Pierre Dansereau : Cette phrase touche réellement à ce que j'ai fait. Pour moi, en effet, le terrain est toujours dans une immersion physique et sensorielle, une confiance qu'il faut donner au corps. Lorsque j'ai débuté, les physiciens s'immergeaient dans les recherches nucléaires, les biologistes développaient la biologie moléculaire - ce sont les deux plus grandes découvertes du siècle dernier, nul ne songerait à le nier - seulement, ces recherches de pointe monopolisaient les crédits et l'attention des étudiants, nous allions à contre-courant en soulignant l'importance du «terrain ». Je ne nie pas un instant les grands bénéfices à tirer de l'Internet et des nouveaux moyens de détection à notre disposition, à commencer par les satellites. Mais être sur place, respirer, sentir, voir, toucher ce qui nous entoure est irremplaçable, nous avons besoin de l'odorat, du toucher, de la vision.

4 1973. La Terre des hommes et le paysage intérieur. Éditions Léméac, Conférence Massey, Radio-Canada, p. 58.

\section{A.P. : D'où vous vient cette sensibilité ?}

Pierre Dansereau : Ça remonte à mon enfance, exceptionnellement heureuse, qui m'a donné pour le restant de mes jours la confiance qui me permet d'être indifférent aux approbations, qui ne viennent pas très vite! C'était en Gaspésie, à l'âge de 5 ans, 6 ans. Je me roulais dans les cailloux, je courais sur la plage pour m'envoler comme les goélands. J'ai toujours fait cela. Et quand j'ai été confronté aux exigences de la science, à l'austérité de la science certains de mes professeurs avaient l'air de me dire : «Ne vous roulez pas dans la boue, ne vous roulez pas parmi les plantes, ne prenez pas trop de plaisir à la beauté des choses, ce n'est pas ça la mission du scientifique ; la mission du scientifique, c'est non pas d'aimer les fleurs mais de les décrire adéquatement, d'apprendre à les nommer, de savoir qui les a découvertes, qui a observé que la violette pouvait projeter ses graines? etc. » Moi je veux voir la violette, projeter ses graines, je veux y toucher, je veux voir jusqu'où ça va. Et surtout, peut-être, le besoin que j'ai toujours eu et qui me sert assez bien actuellement, c'est celui de la continuité, de ne pas dire que tout ce qui est passé avant ne compte plus. Au contraire, on bâtit, for better or for worst, sur le passé; on est qui on a été et, si on espère s'enrichir, ce n'est pas en détruisant ce qu'on a acquis.

Ce que j'avais à offrir à mes élèves, c'était justement ma subjectivité et certaines possibilités de perception. Je n'aurais pas pu être ornithologue, je suis sourd depuis longtemps, et les chants des oiseaux, je n'ai jamais pu les identifier. Les mathématiques? Je ne comprends rien aux chiffres. Est-ce que, à cause de ces handicaps, je ne peux pas être un scientifique? J'ai constaté souvent que je percevais les choses qui m'entouraient beaucoup mieux que les gens qui m'accompagnaient, j'ai du talent pour cela, je tâche de m'en servir aussi longtemps que je pourrai...

A.P. : C'est tout de même peu courant pour un scientifique! Et ce - au risque de me répéter - d'autant plus que vous étendez cette perception à tous les êtres vivant sur cette planète, de la flore à l'homme...

Pierre Dansereau : La présence de l'autre est à la fois une contradiction et un enrichissement. Ça commence d'ailleurs avec l'étude des plantes. J'ai fait ma thèse sur ces plantes admirables que sont les cistes de la garrigue méditerranéenne. Je me suis appliqué à mesurer des milliers et des milliers de feuilles, pour voir le degré de pubescence, la coloration des fleurs à l'intérieur d'une espèce, entre deux espèces. Est-ce que cette différence est assez grande pour qu'on en fasse une variété, ou seulement une forme? Une variété, ou encore une nouvelle espèce? Quel est l'ordre de grandeur dans lequel on travaille? Qu'est-ce qui ne frappe pas l'œil à première vue? Il faut d'abord physiquement se pencher sur son sujet. J'avais fait une promenade avec deux charmantes religieuses dans une forêt et il y avait des euphraises. 
Je me suis mis à genoux pour les cueillir et sœur Sainte Catherine a dit : «Oh ! Il faut y apporter une grande dévotion! » C'était juste! J'éprouvais une certaine dévotion pour cette plante toute petite que j'allais voir d'assez près pour distinguer les rayures violettes qu'il y avait sur la corolle blanche. Et puis celle-ci, qui avait plus de rayures : est-ce que c'était une autre espèce? Cet engagement visà-vis de ce qu'on appelle maintenant la biodiversité. . .

N.B. : Pouvons-nous prolonger un peu sur le terrain de l'éthique, qui est arrivée dans votre parcours à un moment donné. Comment s'est fait cette ouverture?

Pierre Dansereau : Comme vous l'avez compris, il y a eu d'abord l'amour des plantes, le plaisir de les toucher, j'avais trouvé ça dans la littérature française. Ils n'ont pas beaucoup le sens de la nature, les Français, mais j'avais lu à 15 ans Maurice de Guérin ${ }^{5}$, qui à un moment donné embrasse un arbre; cela m'avait beaucoup nourri. Embrasser un arbre! Alors, si j'aimais les arbres, si j'aimais les plantes, cela devait aussi inclure l'herbe à puce, les orties, les plantes vénéneuses, les plantes qui se défendent contre nous... embrasser tout ce monde végétal, animal - les écureuils dans la cour, les chevaux, j'ai fait beaucoup d'équitation quand j'étais jeune et le «partenariat » avec mon cheval blanc quand j'allais dans la montagne l'hiver et qu'il y avait des hiboux sur les branches des arbres, c'était presque de la symbiose.

Le passage vers " 1 'humain » était tout naturel. Issu d'une famille "à l'aise », heureuse, à 20 ans j'avais besoin de changer le monde, et je résolus de faire partie de ceux qui utiliseraient les moyens dont ils disposent pour le faire. À 18, 19 ans, je me suis engagé comme matelot sur un bateau dans l'Arctique et l'année suivante dans les tropiques, j'ai approché de près certaines formes de misère. Je me rappelle un épisode en particulier où j'étais en fond de cale à pelleter du charbon avec ce que l'on peut appeler un hairy ape, un singe velu, et qui était là, torse nu, poilu, et qui pelletait deux fois plus vite que moi et qui s'arrête à un moment donné, et qui dit "You know, Pete, if you ain't got no education, you ain't got nothing »«Si t'as pas d'éducation, $t^{\prime}$ as rien ». J'aurais voulu l'enregistrer. Il m'a fait un long discours sur les beautés de l'éducation, dont il avait été privé. Cet épisode m'a brûlé pendant longtemps. Je me suis dit : «Eh bien moi, qui ai de l'éducation, j'ai le devoir, l'obligation de me servir de tous mes moyens. »

A.P. : C'est une belle transition pour parler de votre engagement personnel, de la nécessité de ce que vous appelez en 1991 "l'austérité joyeuse », c'est-à-dire le «consentement à de multiples contraintes que nous

\footnotetext{
${ }^{5}$ Guérin, M., 1922. Trebution, Guillaume S, Journal lettres et poèmes, Paris, Librairie Victor Lecoffre, 27 e édition.
}

devrions nous imposer avant que nous ne soyons obligés de nous y soumettre ${ }^{6} » .$. Pouvez-vous développer?

Pierre Dansereau : «L'austérité joyeuse » est une prédisposition au partage des surplus que nous avons; or notre société est plutôt dans la surconsommation. «L'austérité joyeuse » ne saurait commencer qu'avec l'identification de ce qui nous entoure, la conscience du milieu, la présence aux objets du quotidien, c'est à cela que j'ai beaucoup travaillé avec mes étudiants. Je leur faisais faire par exemple une carte, à l'échelle ou à peu près, de leur logement, en pointant tout ce qu'il contenait et en les faisant remonter jusqu'aux agents responsables du bienfait dont ils bénéficiaient : une table en chêne blanc massif de la tante Alice qui l'avait achetée en Californie à un artisan; une pomme venant de Saint-Hilaire, une tasse de café sur la table, résultat de quatorze opérations, entre la graine semée par des esclaves colombiens ou cubains, le soin de la plante, la récolte, le transport, la torréfaction, la mise sur le marché, l'emballage, la préparation... Vous, en tant que consommateur, vous êtes au bout de cette chaîne; à qui êtes-vous redevable? À qui devez-vous être reconnaissant?

Au-delà des surplus, quelle aide devons-nous apporter à ceux qui manquent de l'essentiel, à savoir pour se nourrir, se vêtir, se loger, s'éduquer... Sur les besoins, j'ai fait un petit schéma du gâteau de l'environnement dans lequel les besoins physiologiques, psychologiques, sociaux, etc., sont reconnus comme des catégories différentes avec quatre niveaux de satisfaction, depuis la privation jusqu'au surplus.

N.B. : Pensez-vous qu'on va pouvoir arriver à cette identification de ce qui nous entoure, pour tendre vers une plus grande « austérité joyeuse » ou, comme diraient d'autres auteurs, une "simplicité volontaire »?

Pierre Dansereau : Je crois que si ça ne commence pas dans l'enfance, ça n'a pas beaucoup de chances de se réaliser; il faut que l'éducation fonctionne à plein ; le rôle peu efficace à ce point de vue-là des grands médias n'est pas encourageant. Actuellement, ils flottent presque tous au niveau de la surconsommation, de l'économie néolibérale, de l'affaiblissement du rôle et du contrôle de l'État, d'une privatisation qui s'est élevée à l'état de religion ou peu s'en faut, alors on s'en va dans la mauvaise direction. Mais j'ose espérer malgré tout, non seulement parce que je suis venu au monde optimiste, avec un parcours heureux, mais aussi parce que j'ai la foi.

N.B. : Vous parlez du rôle peu efficace des médias par rapport à la situation assez problématique que l'on vit actuellement, pouvez-vous dire deux mots sur le rôle de la science et le rôle des scientifiques par rapport à l'identification de solutions et à leur mise en œuvre?

\footnotetext{
${ }^{6}$ Dansereau, P., 1991. L'Envers et l'endroit : le besoin, le désir et la capacité, Québec, Musée de la Civilisation.
} 
Pierre Dansereau : Les scientifiques d'il y a trente ou quarante ans étaient presque absents de la scène publique. Mais qui peut interpréter les choix qui nous confrontent aujourd'hui, devant la nouvelle capacité des opérations transgéniques, par exemple? On a besoin d'informations compétentes pour aboutir à une éthique ; si on a un gadget, faut-il s'en servir sans discernement? La réponse est «non »! Le fait que nous ayons augmenté notre puissance ne nous autorise d'aucune façon à intervenir sans nous poser des questions d'ordre éthique, d'ordre moral. Et je crois qu'il n'y a pas beaucoup de Hubert Reeves pour aller très loin dans l'interprétation des moyens nouveaux et plus forts dont nous disposons...

A.P. : Dans votre film sur la favela brésilienne, j'ai été frappée par cette image : vous êtes sur une plage et vous évoquez sans états d'âme une plante qui va disparaître puisqu'on ne peut pas et on ne doit pas interdire aux hommes l'accès à ce lieu superbe. Votre priorité va définitivement vers l'homme maintenant?

Pierre Dansereau : Oui, tout à fait. Dans cette scène, on voit aussi ces beaux enfants, presque nus, qui se renvoient la balle, c'est plus important que la plante... Ce qu'un homme peut faire... Je pense par exemple à Nelson Mandela, qui est pour moi le plus grand homme du vingtième siècle, qui a évité le bain de sang que tout le monde prévoyait, qui, au lieu de la vengeance, du ressentiment, a fait triompher la coopération, pour l'appeler par son nom, l'amour.

N.B. : Alors, peut-être la dernière question quej'aimerais vous poser : quelles sont les recherches essentielles qui vous animent encore aujourd'hui ? Quelles questions vous posez-vous? Au fond, que cherchez-vous encore à décoder?

Pierre Dansereau : Il y a longtemps que j'ai trouvé le titre de ma biographie, c'est Projets inachevés. Comme vous le savez peut-être, j'ai un grand projet en cours qui est celui sur la végétation à très petite échelle. Une petite échelle, c'est une grande superficie représentée par une petite superficie. Nous travaillons depuis douze ans peut-être, Daniel Garneau et moi, à faire cette cartographie. C'est très avancé, la recherche est presque complètement terminée. Le résultat final devrait servir dans les écoles et un peu partout à projeter l'image de la végétation de la Terre avant la venue de l'Homme. Cela ne représente d'aucune façon l'état actuel de la planète, mais celui que nous pensons avoir existé avant l'Homme.

A.P. : En quoi votre approche sur le terrain vous aidet-elle dans ce travail d'analyse de données?

Pierre Dansereau : Grâce à la cartographie numérique, nous avons une documentation à notre disposition incomparablement plus riche et plus accessible qu'il y a vingt ou trente ans. Néanmoins et pour ma part, j'ai une certaine réserve vis-à-vis de tout ce qui est reconstitué par des manipulations numériques. Rien ne vaut une visite sur le terrain et, heureusement, Daniel et moi nous avons pas mal voyagé et il y a des certitudes que nous pouvons avoir parce que nous sommes allés dans ces endroits ou alors nous avons des documents qui sont très clairs et nets, comme des photographies, par exemple. Mais nous avons encore du travail à faire pour analyser toutes nos données.

A.P. : J'aimerai conclure, car malheureusement il le faut bien, sur cette « déférence à l'autre » qui a été votre fil conducteur tout au long de votre vie, tout d'abord au niveau de votre vie de scientifique, mais aussi de votre vie personnelle, d'ailleurs intimement liées si j'ai bien compris votre parcours, puisque vous êtes un scientifique «engagé ».

Pierre Dansereau : C'est vrai, le contact et l'échange nous rendent plus conscients des choses que nous ne savions pas et que nous pouvons apprendre. Une autre vision d'un problème ou d'un objet nous aide à confirmer et à explorer nos propres limitations. Moi, ça m'est arrivé d'entendre des mathématiciens parler, je ne comprenais pas le cheminement, mais j'arrivais à assimiler ce qu'ils avaient à dire lorsqu'ils en venaient à leurs conclusions. Quand Hubert Reeves dit que nous voyons des étoiles qui n'existent plus, ça a été une révélation pour moi. Je n'arriverais probablement pas à le suivre dans les étapes de la découverte mais cela n'a pas d'importance.

A.P. : Ce qui est important, c'est ce que cela a provoqué chez vous?

Pierre Dansereau : Oui et je suis curieux de rencontrer des gens. Quelques-uns, je peux les suivre depuis les prémisses jusqu'à la conclusion. D'autres, j'ai du mal à m'adapter aux prémisses, à les saisir, mais quand vient la conclusion, ça cadre avec ce que je sais déjà, ça s'intègre dans la mosaïque de ma compréhension.

Et quant à ma vie personnelle, cette « déférence à l'autre » a été une constante. Je pense que ce qui me préoccupe peut-être le plus personnellement, intimement, si je peux dire, c'est une double définition de la dimension spirituelle. Tout en demeurant attaché à l'Église catholique, je suis décidément sur le portique, et non pas dans le chœur, j'accepte, je cherche ma propre interprétation du message de Jésus, tout en me mettant à distance des cinquante dogmes... Cela ne m'inspire pas de l'antagonisme, c'est simplement des croyances dont j'ai vécu, comme tous ceux de ma génération, et dont je m'éloigne et je cherche une certaine formulation, pas du tout dans l'angoisse, absolument pas. J'essaie actuellement de décrire ce processus. J'en viendrai peut-être à bout. 


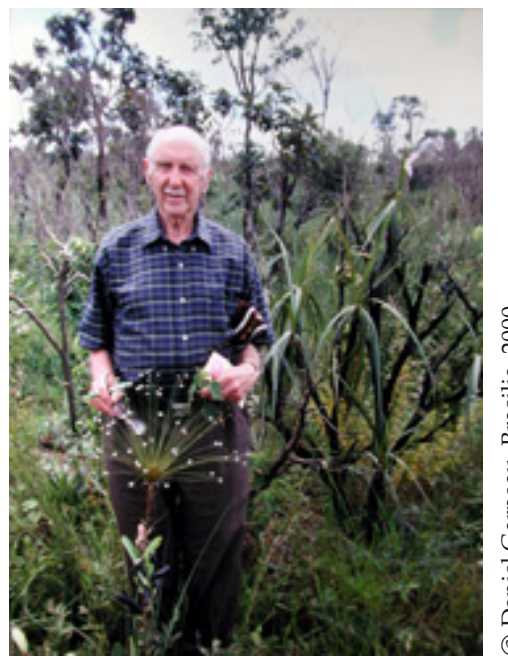

\section{Biographie}

Pierre Dansereau est né à Montréal en 1911. Docteur ès sciences de l’Université de Genève (1939). Professeur aux universités de Montréal, du Michigan (Ann Arbor), de Columbia (New York), du Québec à Montréal [professeur émérite] ; professeur invité à une douzaine d'universités ; chercheur, notamment aux jardins botaniques de Montréal, de Rio de Janeiro et de New York.

- Auteur de nombreuses contributions scientifiques (taxonomie ; écologie végétale, urbaine et humaine ; biogéographie; aménagement du territoire) et de plusieurs livres, dont Biogeography: an ecological perspective (1957), La Terre des hommes et le paysage intérieur (1973), EZAIM : Écologie de la zone de l'aéroport international de Montréal (1976), Harmonie et désordre dans l'environnement canadien (1980), L'Envers et l'endroit : le besoin, le désir et la capacité (1994).

- Membre de la Société royale du Canada, compagnon de l'Ordre du Canada, chevalier et grand officier de l'Ordre national du Québec, membre du Panthéon de la science et de l'ingénierie canadiennes; dix-sept doctorats d'honneur lui ont été décernés.

- Pionnier de l'écologie, sommité mondiale, cité dans l'Encyclopaedia Britannica.

- Films consacrés à Pierre Dansereau : Quelques raisons d'espérer (Fernand Dansereau, ONF, 2001) ; L'Homme de toute la terre (André Larochelle, 2002). 